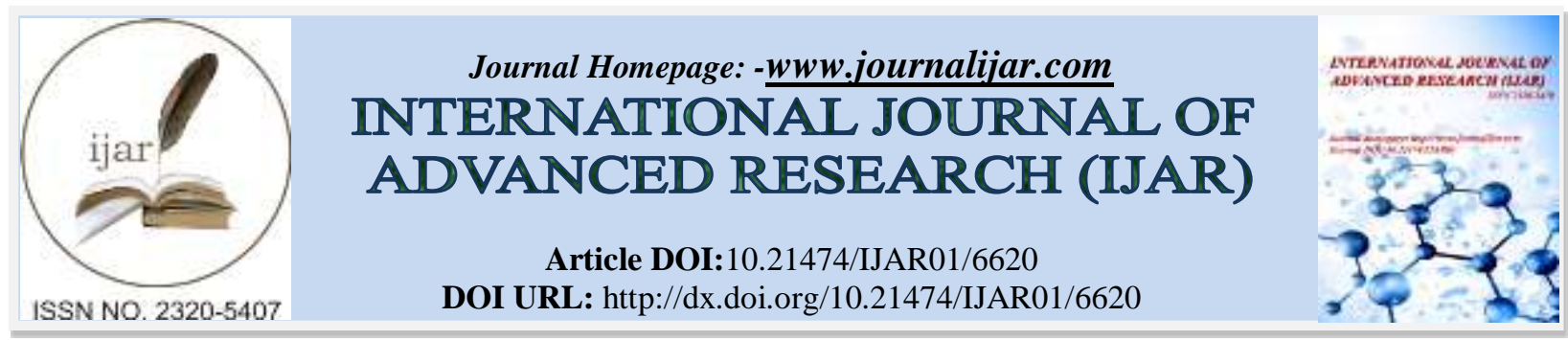

RESEARCH ARTICLE

\title{
REVIEW OF INCIDENCE OF CARCINOID LUNG TUMORS IN IRAQ.
}

Dr. waleed M. hussein M. S $^{1}$, Dr. Abdul-Razzaq N. Rashied ${ }^{2}$ and Dr. sadoon. H. Al-Hayazie ${ }^{3}$.

1. FIBMS(th.c.v.s)PACs.,MRcs.,FRCS(Glasgow).professor and consultant of cardiothoracic and vascular surgeon.chairman of the scientific Council of thoracicand cardiovascular surgery.

2. MBCHB.FICMS cardiothoracic and vascular surgeon.

\section{Manuscript Info}

Manuscript History

Received: 23 December 2017

Final Accepted: 25 January 2018

Published: February 2018

Keywords:-

Carcinoid lung tumors, atypical carcinoid, collapse lung, resection.

\section{Abstract}

Background: Carcinoid lung tumors are rare tumor which tend to be slow growing. They are one type of neuroendocrine tumors, they have two type, typical (low grade) and atypical (intermediate grade). $1.2 \%$ of primary lung tumors are carcinoid. The average age of people affected is 40-50 years for typical subtype while atypical have been reported in virtually every age group. Cough, dyspnea, hemoptysis and recurrent chest infection are the main presenting symptoms. Chest x-ray, CT scan of chest and bronchoscopy are the main tools in the diagnosis of carcinoid lung tumor. Management was mainly surgical with resection of the affected lobe or lobes of the lung obiective: the study is planned to review the incidence of carcinoid lung tumor, methods of diagnosis and outcome of surgical treatment.

Study design: retrospective and comparative study

Setting: Department of Thoracic Surgery of Al-shaheed Ghazi hariri Hospital, Medical City Teaching Complex, Baghdad, Iraq

Patients and methods: review of clinical record and surgeon note of 20-patients with carcinoid lung tumor during 18-years (1996-2013). Collecting information relevant to patients variable with regard to age, sex, presenting symptoms, radiographic findings (chest x-ray and CT scan) bronchoscopic finding, pre-operative preparation, post-operative course and the histopathology of the resected specimens.

Results: 13 patients out of 20 (65\%), were under the age of 40 year. 11 patients out of $20(55 \%)$ were female. Cough and shortness of breath are the most common presenting symptoms. All of patients have a typical bronchoscopic findings with cherry-red-coloured, smooth, polypoid, vascular tumor that bleeds easily and profusely. All the patients were managed surgically with formal postero lateral thoracotomy and resection of affected part of lung and there was no significant post-operative complication.

conclusion: Early detection of bronchial carcinoid tumor with the use of Endobronchial ultra Sound EUs or future tumor markers, a more conservative surgical procedure such as sleeve or wedge resection, can be adopted with the hope of saving more functioning lung tissue.

Copy Right, IJAR, 2018,. All rights reserved. 


\section{Introduction:-}

Carcinoid tumors Are rare tumors which tend to be slow growing. They may not cause any symptoms for several years. Most of these tumors occur in people over the age of 60 years. They are one type tumor of the neuroendocrine system. Most carcinoid tumors are found in digestive system, but they can also develop in lung, pancreas, kidney, ovaries or testicles (1).

\section{Carcinoid tumors of lung:-}

It arises from Kultchisky Amine precursor uptake Decarboxylation(APUD) cells in the bronchial epithelium $(2,3)$. The bronchopulmonary carcinoid tumor classified on the basis of WHO.

guidelines 2004 and that describe four subtype of bronchopulmonary neuroendocrine tumor which are typical (low grade), atypical (intermediate grade), large cell neuroendocrine carcinoma and small cell lung carcinoma (4).Both typical and atypical carcinoid tumors consist of small nests or interconnecting trabeculae of uniform cells separated by a prominent vascular troma and numerous thin-walled blood vessels $(2,3)$. The difference between typical and atypical bronchopulmonary carcinoid tumor illustered in table no.1

\begin{tabular}{|l|l|l|l|l|}
\hline & location & Nodal metastasis & $\begin{array}{l}\text { Gross pathological } \\
\text { examination }\end{array}$ & WHO criteria \\
\hline $\begin{array}{l}\text { Typical carcinoid } \\
\text { tumor }\end{array}$ & central & rare & $\begin{array}{l}\text { White or gray, with } \\
\text { minimal evidence of } \\
\text { necrosis } \\
\text { hemorrhage }\end{array}$ & $\begin{array}{l}\text { or } 2 \text { Size }>0.5 \mathrm{~cm} \\
\text { Lack of necrosis }\end{array}$ \\
\hline $\begin{array}{l}\text { Atypical carcinoid } \\
\text { tumor }\end{array}$ & peripheral & frequent & $\begin{array}{l}\text { White-gray;on } \\
\text { section,tan,pink,red, } \\
\text { or yellow-brown }\end{array}$ & $\begin{array}{l}2-10 \text { mitosis per } \\
\text { mm2 } \\
\text { Size }>0.5 \mathrm{~cm} \\
\text { Necrosis }\end{array}$ \\
\hline
\end{tabular}

As neuroendocrine tumors, carcinoids are capable of producing a variety of biologically active peptides and hormones, including serotonin, adrenocorticotropin hormone (ACTH), antidiuretic hormone (ADH), melanocytestimulating hormone $(\mathrm{MsH})$, and others $(3,5,6)$.Excess serotonin production has been implicated in the development of carcinoid syndrome. This syndrome is characterized by a constellation of systems and manifests when vasoactive substance from tumor enter the systemic circulation escaping the hepatic degradation. This is the case when carcinoid tumors metastasize to the liver or they arise in the bronchus. These tumors release too much of the hormone serotonin and several other chemicals that cause the blood vessels to open (dilate) (2,7).Ectopic production of ACTH and Cushing syndrome have been reported in association with typical and atypical carcinoid tumors. Although less than $1 \%$ of pulmonary carcinoid tumors produce Cushing syndrome, it is the second most common neuroendocrine syndrome produced by these tumors. In addition, these tumors are responsible for development of about $1 \%$ of cases of Cushing syndrome. When a patient is found to have an ectopic source of ACTH production, the lesion is generally a pulmonary neoplasm of same type $(3,5,6)$. The syndrome of inappropriate AVP (arginine vasopressin) secretion or syndrome of inappropriate secretion of $\mathrm{ADH}$ (SIADH) can be produced by pulmonary carcinoid tumors, although it more commonly is associated with small cell lung carcinoma. The production of excess circulating AVP creates hypothermia secondary to water retention.

Patients present with weight gain, weakness, lethargy, and mental confusion and, in sever cases, can develop convulsion and coma $(3,5,6)$.

\section{Diagnosis:-}

Laboratory studies:-

Assay of specific neuroendocrine substances like 5-hydroxyindoleacetic acid(5-HIAA),ACTH,MSH,growth hormone and others.

\section{Imaging studies:-}

Chest radiographyA Changes associated with bronchial obstruction include persistant atelectasis, consolidation secondary to pneumonia, and of branchiectasis and hyperinflation changes (6). 


\section{Computed tomography scan:-}

High-resolution cr scan is the best type of CT examination for evaluation of a pulmonary carcinoid tumor. It may reveal nodules or masses that not well visualized on plain chest radiograph by virtue of their small size or their position, such as t located in a retrocardiac position.

Magnetic resonance imaging MRI generally provides information similar to that of CT studies. Dynamic MRI may be a useful complimentary examination in selected cases(13).

Positron emission tomogrophy Positron emission tomography (PET) studies utilize the fact that malignant cells possess a higher metabolic activity rate than do healthy cells. A tagged glucose molecule, FDG (2-fluorine-18) fluoro 2 deoxy-D glucose), is administered, and metabolic analysis of this substance within the ells of the imaged organ system or the whole body is conducted. PET scan appears to have a considerable sensitivity and specificity for the identification of malignant lesions(14).

\section{Diagnostic Procedures:-}

\section{Bronchoscopy:-}

Because of majority of bronchopulmonary tumors are centrally located, they are amenable to bronchoscopic evaluation. It is difficult to distinguish typical from atypical carcinoid tumor with small biopsy typically obtained by flexible bronchoscopy (4).The characteristic bronchoscopic appearance of a cherry-red coloured, smooth polypoid, vascular tumor that bleeds easily and profusely (2).Hemorrhage is the main complication previously but now its rare, however the risk of hemorrhage can be reduced by administration of epinephrine solution before biopsy. Incase of significant hemorrhage which is difficult to control, a neodymium: yttrium-aluminum-garnet (Nd:YAG) laser is helpful (4).

Peripheral carcinoid lesion can be evaluated by CT-guided percutaneous transthoracic needle biopsy and video assisted thoracoscopic biopsy(4).

\section{Management:- surgical therapy:-}

Surgical resection is the primary mode of therapy for carcinoid tumors of the lung. A variety of forms of resection have been util successfully and with excellent long term results.

Endobronchial management Typical bronchopulmonary carcinoid tumor which is strict endoluminal lesion and no evidence of lymph node invasion can be treated with flexible bronchoscopy and cryotherapy. Also endobronchial resection should be reserved for patient who are not amenable to surgical intervention Bronchoscopic resection using an Nd YAG laser with or without photodynamic therapy also has been utilized in selected cases. As yet, these forms of treatment have been reserved for pre resection reduction of intrabronchial tumor mass or for palliative management of airway obstruction in cases in which the patient was considered otherwise inoperable $(20,21)$.

\section{Patients and Methods:-}

This is a retrospective and comparative study of 20 patients with pulmonary carcinoid tumors, who were admitted and surgically treated at the Department of Thoracic Surgery of the Al- shaheed Ghazi Al-hariri Hospital, Medical City Teaching Complex during 18-years period (1996- 2013 The medical records plus surgeon note of these patient with the diagnosis of carcinoid lung tumor, were reviewed, collecting information relevant to patients variables with regard to patients age, sex, presenting symptoms, radiographic findings (chest x-ray and computed tomography), typical bronchoscopic appearance, pre-operative preparation, operative findings, post-operative complications and results of histopathology of the resected specimen. Rigid bronchoscopy was used almost exclusively in all patients and the typical bronchoscopic appearance of the tumor and its exact location were documented. All patients after a full pre-operative preparations including basic, specific investigations and blood preparations, underwent formal postero-lateral thoracotomy and the collapsed or the diseased lobe or lobes or lung was resected in the classical way in terms of ligation and division of the arterial supply and venous drainage of the affected area, then division and securing bronchus and finally testing for air leak, inserting two intercostal drains and closing the incision in layers. All of our patients had uneventful post-operative course, patients were discharged home in a good condition with no recurrence was observed in the following years in these patient attending regular follow up until recently only two patients were seen and followed. 


\section{Results:-}

Results of 20 patients with carcinoid tumor, who were admitted and surgically managed at Department of Thoracic Surgery of AHshaheed Ghazi Al-hariri Hospital, eleven of them were female constituting (55\%) and the rest (ninepatients) were male constituting $(45 \%)$. The youngest patient was a 25 -years old women, and the oldest was a 58 years old man. Most of the patients (8 patients (40\%)) fall between 2030 years old. The distribution of our patients as regard their age and sex is shown in figure (4).

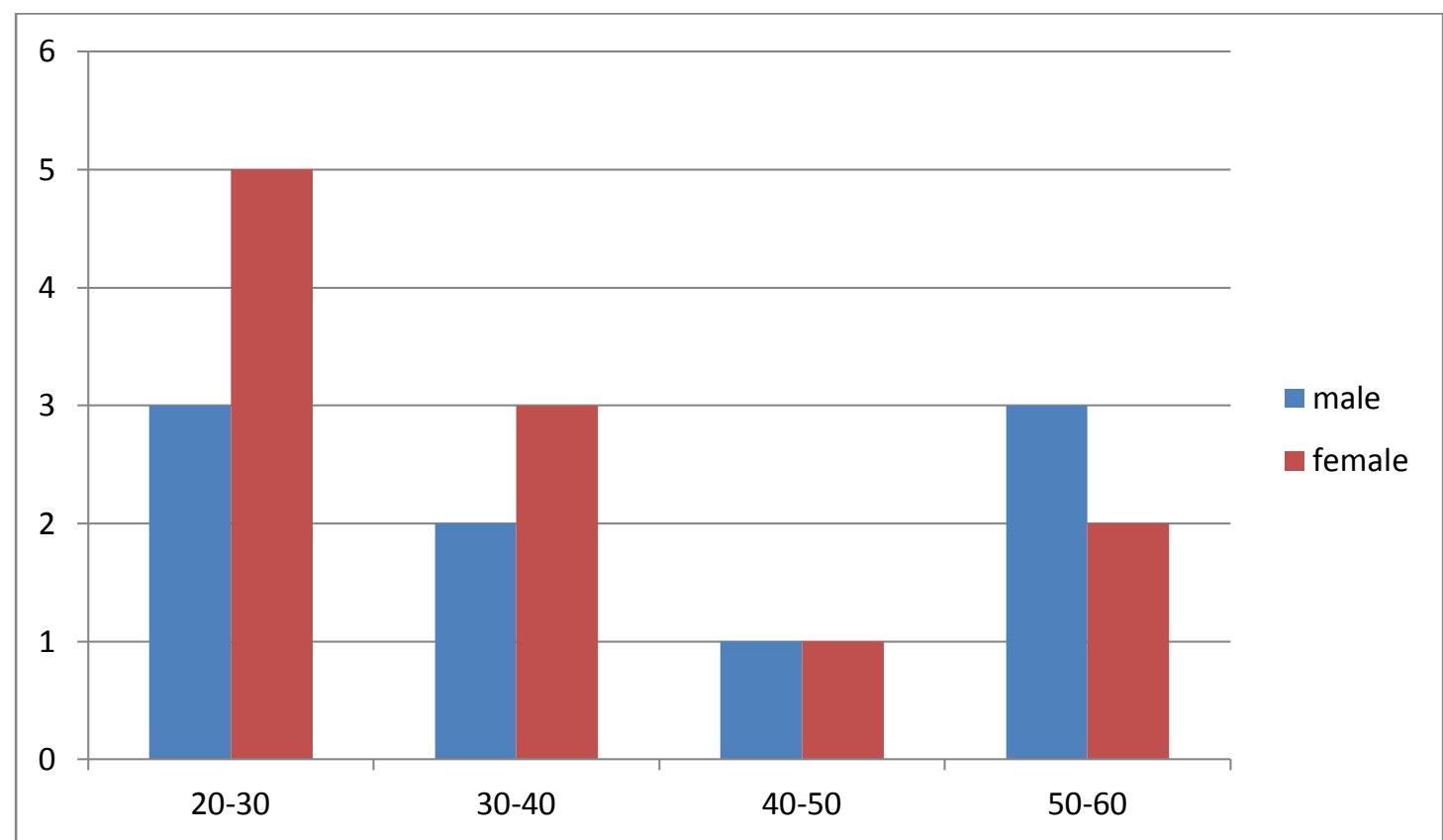

Figure 4:- age and sex distribution Fifteen patients presented with dry irritative cough constituting 75\%). Easy fatigability and shortness of breath were seen in eleven patients (55\%). Recurrent chest infection was seen in six patients (30\%) only. Hemoptysis was seen in five patients (2596) only. Carcinoid syndrome presented with attack of (flushing, sweating, diarrhea, fever) was so rare that it is seen in only one patient (5\%) The distribution of the above mentioned clinical presentation is shown in figure (5).

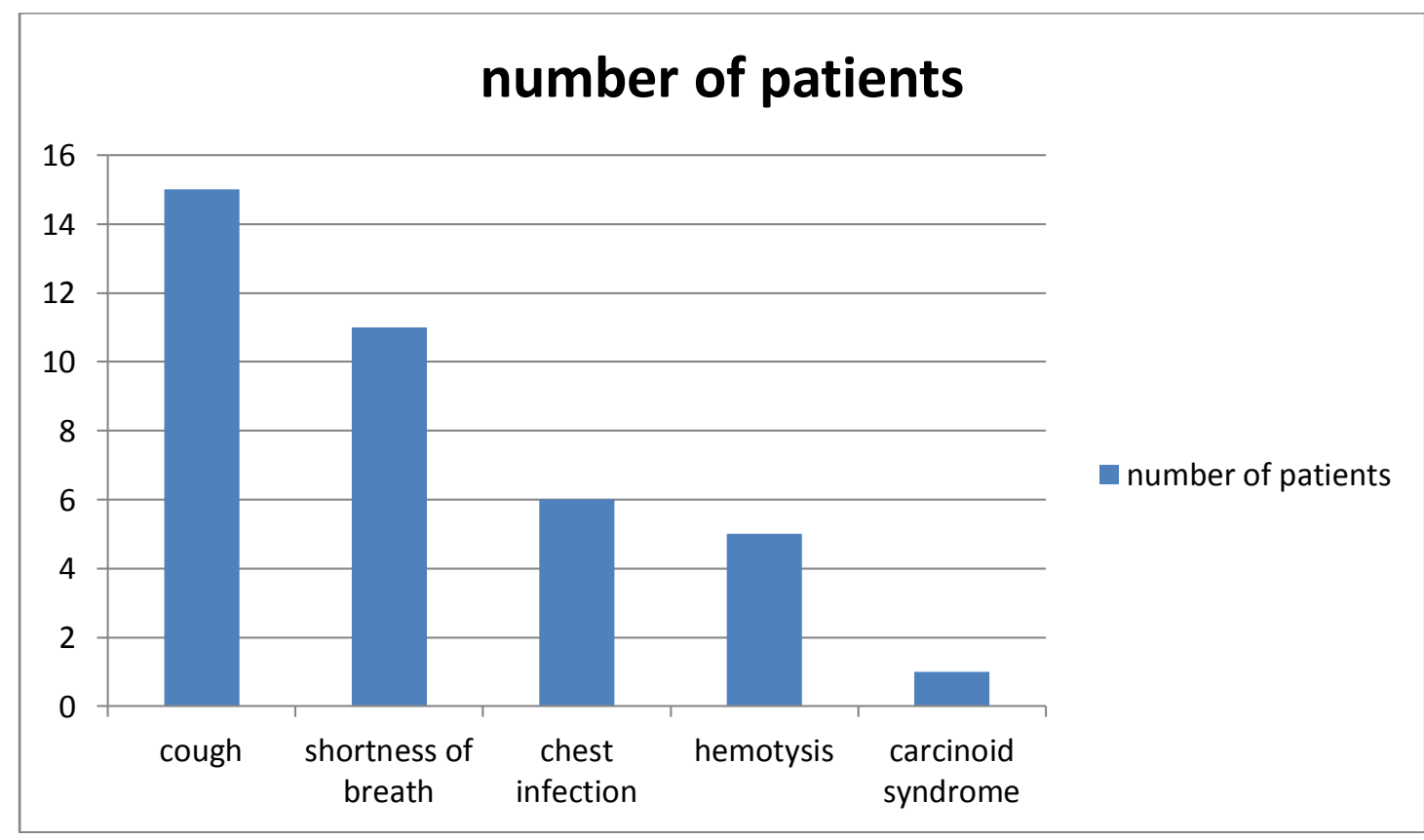


Chest radiography was performed to all patients, as the primary imaging modality and the radiographic appearance of these patients is illustrated in table (2).

\begin{tabular}{|l|l|}
\hline findings & No.(\%) \\
\hline Collapsed left lung & $7(35 \%)$ \\
Collapsed left upper lobe & $3(15 \%)$ \\
Collapsed right middle and lower lobe & $3(15 \%)$ \\
Collapsed right upper lobe & $2(10 \%)$ \\
Collapsed right lung & $2(10 \%)$ \\
Collapsed right lower lobe & $2(10 \%)$ \\
Collapsed right middle lobe & $1(5 \%)$ \\
\hline
\end{tabular}

CT scan was done to all patients and showed an endobronchial mass localized to a lobe or lobes or main bronchus, which coincides with the radiographic findings. The typical bronchoscopic finding was documented in all cases and its typical appearance of cherry-red coloured, smooth, polypoid, vascular tumor that bleeds easily on touch, was seen in almost all patients. Biopsy was done in the first two patients followed by sever bleeding, that was controlled with difficulty. All these patients underwent formal resection of the affected lobe, lobes or lung after a full preoperative assessment making these patients in an optimum condition prior to surgery. The resected specimens were sent for histopathological study. The modalities of the surgical resection are illustered in table (3).

\begin{tabular}{|l|l|}
\hline Type of surgery & No.(\%) \\
\hline Left pnemonectomy & $7(35 \%)$ \\
Left upper lobectomy & $3(15 \%)$ \\
Right middle and lower lobectomy & $3(15 \%)$ \\
Right upper lobectomy & $2(10 \%)$ \\
Right pnemonectomy & $2(10 \%)$ \\
Right lower lobectomy & $2(10 \%)$ \\
Right middle lobectomy & $1(5 \%)$ \\
\hline
\end{tabular}

All of our patients ran an uneventful post-operative course with only wound infection in two patients treated conservatively by culturing of the wound discharge and accordingly antibiotic given. The obtained histopathological report in 19- patients confirmed that the mass is typical of carcinoid tumor with tumor free resected margin. In only one female patient with attack carcinoid syndrome prior to surgery, the histopathological report turned out to be an atypical carcinoid of intermediate grade, who was refered to the oncologist post-operatively for radiotherapy. The patient defaulted during the follow up period. Two of our patients are currently followed up complaining of wheezy chest during winter time only.

\section{Discussion:-}

Carcinoid tumors are rare entity but still encountered in the Thoracic Surgery Department. They are uncommon lowgrade malignant tumor which are most commonly seen in the gastrointestinal tract while pulmonary carcinoids come in the second most common site (25).

our small number of patients confirmed that it is an uncommon condition, and this coincides with other study by Akiba et all(26).who reported 32 patients over 24-year (1965-1989) only. Female patients were affected more than male patients in our study 11:9, with a ratio $1.25: 1$ and this coinsides with other study (27).However, it is in contradiction to other studies, reporting a higher incidence among male patients (26), where as Hamid et a reported equal sex incidence.

The majority of our patients 13 - patient $(65 \%)$ seen between $20-40$ years of age which is similar to other studies $(28,29)$.

Cough, dyspnea and hemoptysis, were the most common presenting symptoms and similar symptoms reported with other studies $(28,29)$. 
Chest radiography was performed to all patients. All of them have abnormal findings such as collapsed lobes or collapsed lung where as Hamid et al (28)described an abnormal chest radiography in only 16 out of 21 patients (76.2\%). CT scan findings showed an intrabronchial mass localized to lobar or main bronchus associated with collapsed consolidation of the affected area of lung with no pleural effusion. Bronchoscopy under general anesthesia was used in all patients as the main diagnostic tool with the appearance of the typical findings of the carcinoid tumor obstructing left main bronchus or left upper lobe bronchus in 10-patient (50\%) out of 20-patient, Other studies showed that the right main bronchus was predominantly affected Ronchod reported that the right middle lobe is the most commonly affected(28). Bronchial wash unfortunately, failed to confirm the presence of malignant cells and this is in agreement with other studies $(28,30)$.

Biopsy which was done in two patients only confirmed the presence of carcinoid tumor, but was unfortuntly followed by sever bleeding, that was controlled with difficulty, in contradiction to study conducted by hamid et al (28).in which endobronchial biopsy was the main stay of diagnosis

Surgery was the main modality of treatment with pneumonectomy which was performed in nine patients out of 20 patients (45\% It includes left pneumonectomy in seven patients and right pneumonectomy in two patients, followed by lobectomy in eight patients and bilobectomy in three patients while lobectomy was the main surgical procedure in other work $(31,32)$.In contrast to our radical resection, Ismail et al(31) adopted the policy of paranchyma sparing or tissue-saving operations as the treatment of choice for carcinoid in 29 out of 83 patients (349\%), whereas Elhassan's personal experience in the surgical management of carcinoid tumor consisted of bronchotomy or sleeve resection of the bronchus whenever possible and lobectomy or pneumonectomy whenever there is tumor extension to the lung paranchyma, if the lesion has caused perminant irreversible pulmonary suppuration and if there was intrathoracic nodal involvement (32).

Harpole et at (23)did not recommended bronchoscopic resection, as this procedure was followed by recurrence of the tumor in two cases of their study. Post-operative complications, were seen in only two patients in the form of wound infection, treated effectively conservatively All of our patients showed complete recovery, with no mortality and only two of them attending for follow up till today. The rest completely disappeared for follow up.

\section{Conclusion:-}

1. Early detection of bronchial carcinoid tumor with the use of EUs or future tumor markers, a more conservative surgical procedure such as sleeve or wedge resection, can be adopted with the hope of saving more functioning lung tissue.

2. Surgery offers the best chance of cure and the most fruitful outcome in patient with carcinoid lung tumor.

\section{Recommendations:-}

1. carcinoid lung tumors, should be always kept in mind in the differential diagnosis of patients with respiratory signs and symptoms (cough, shortness of breath, recurrent chest infection and or hemoptysis)with the imaging findings of atelectasis confined to a lobe, lobes or lung.

2. Introducing Endobronchial ultra-sound (Eus) and arranging workshops to train qualified personnel, so that it can be used as a diagnostic tool.

3. Making the availability of the tumor marker and hormonal assay such as 5-hydroxy indol acetic acid (5HIAA), which is a metabolite of serotonin. Testing urinary level of it is used for diagnosis of carcinoid lung tumors associated with carcinoid syndrome.

4. Documentation is necessary for reviewing cases, research making and planning future line for accurate management.

\section{References:-}

1. http://m.cancer.org/cancer/lungcarcinoidtumor/detaileguide/lung-carcinoid-tumor-what-is-lung carcinoidtumor.

2. Waleed M. Hussen, Abdulameer M. Hussein. Carcinoid lung tumor. The Egyptian Journal of Surgery 2014:33:160-163 .

3. Chong S, Lee KS, Chung MJ. Neuroendocrine tumors of the lung: clinical, pathologic, and imaging findings. Radiographics. Jan Feb 2006,26(1):41-57; discussion 57-8 . 
4. Frank Sellke, Pedro J. Nid, Scott Swanson. Sabiston and spencer's Surgery of chest 8TH edition 2010 22(2):323-28. 5- Rusch VW, Klimstra DC, Venkatraman ES. Molecular markers help characterize neuroendocrine lung tumors. Ann thoracic surgery. 1996:62:798-810 .

5. Meisinger QC, Klein US, Butnor KJ, Gentchos G. Leavitt Bj. CT Features of Peripheral Pulmonary Carcinoid Tumors. AJR Am JRoentgenol. Nov 2010.197(5):1073-80 .

6. Hande KR in Goldman L, Schafer AL, eds Carcinoid syndrome. Cecil medicine. 24TH ed Philadelphia, PA: Saunders Elsevier, 2011.

7. Meade RH. Tumors and cysts of the lung. In: A History of Thoracic Surgery. Springfield, Ill Charles C. Thomas; 1961:175-222.

8. E.Goljan, Rapid review pathology, 2nd ed. Tulsa, oklahoma, USA: Mosby Elsevier. 10. Cote ML, Wenzlaff AS, Philip PA, Schwartz AG. Secondary cancers after a lung carcinoid primary: a population-based analysis. Lung Cancer. Jun 2006;52(3):273-9

9. Choman S. Omer. Chest x-ray made easy.2009;3:27-64

10. M. Bakhshyesh Karam, S. Zahirfad, M.O. Tahbaz, K. Kaynama, F. Tolau, H.Jabari Darjani et al. Bronchial carcinoid tumors clinical and radiological study. Iran J. Radiol. June 2005:2(3,4):111-16 .

11. Guckel C, Schnabel K, Deimling M. Solitary pulmonary nodules: MR re-evaluation of enhancement patterns with contrast-enhanced dynamic snapshot gradient-echo imaging. Radiology. 1996;200:681-86 .

12. Jindal T, Kumar A, Venkitaraman B, Meena M, Kumar R, Malhorta A, et al. evaluation of the role of [18FlFDG PET/CT and 168GalDOTATOC-PET/CT in differentiating typical and atypical pulmonary carcinoids. Cancer imaging. Jun 152011 11:70-5 .

13. Esfahani AF, Chavoshi M, Noorani MH, Saghari M, Eftekhari M,Beiki D, et al. succeful application of technetium-99m-labeled octreotide acetate scintigraphy in the detection of ectopic adrenocorticotropinproducing bronchial carcinoid lung tumor: a case report. J Med Case Reports. Oct 18 2010,4 323 .

14. Hubalewska-Dydejczyk A, Fross-Baron K, Mikolajczak R. (99m)Tc-EDDA/HYNIC-octreotate scintigraphy, an efficient method for the detection and staging of carcinoid tumours: result of 3 years' experience. Eur J Nucl Med Mol Imaging. 2006.

15. MusiM, Carbone RG, Bertocchi c Branchial carcinoid tumours: a study on clinicopathological features and role of octreotide scintigraphy, Lung cancer 1998 2297. 102.

16. Yendamuri S. Gold D, Jayaprakash V, Dexter E. Nwogu C, Demmy T. Is sublobar Resection sufficient for carcinoid Tumors? Ann Thorac Surg. Jun 232011.

17. Tastepe Al. Kurul IC, Demircan S. long-term survival following bronchotomy for polypoid bronchial carcinoid tumours. European Jornal of Cardio-thoracic surgery 1998 14:575-577.

18. Chughtai TS, Morin JE, Sheiner NM, Wilson JA, Mulder Ds. Bronchial carcinoid- twenty years' experience defines a selective surgical approach. Surgery, Oct 1997;12204) 801-8.

19. Lemaitre J Mansour Z, Kochetkova EA, Koriche C. Ducrocq x, Wihlm JM, Bronchoplastic lobectomy: do early results depend on the underlying pathology A comparison between typical carcinoids and primary lung cancer. Eur J Cardiothorac Surg Jan 2006;3001:168- 71.

20. Ducrocq $\mathrm{x}$, Thomas P, Massard G. operative risk and prognostic factors of typical bronchial carcinoid tumors. Ann Thorac Surg. 1998:65:1410-4 .

21. Harpole DH Jr, Feldman JM, Buchanan S. Bronchial carcinoid tumors: a retrospective analysis of 126 patients. Ann Thorac Surg. Jul 1992;s4f1:50-4 discussion 54-5.

22. Rea F. Binda R, Spreafico G. bronchial carcinoids: areview of 60 patients. Ann Thorac Surg Mar 1989:47 (3):412-4 .

23. Modlin IM, Lye KD, Kidd MA 5 decade analysis of 13.715 carcinoid tumor. Cancer 2003.934-59 .

24. TAkiba, T Naruke. H Kondo, et al:carcinoid tumor of the lungciincopathological study of 32 cases, Jpn J Clin Oncol 1992;22:92-95 .

25. Paladugu RR, Benfield JR, Pak HY, et al. Bronchopulmonary Kulchitzky cells carcinomas: a new classification scheme for typical and atypical carcinoid cancer.1985;55:1303-11.

26. Hamid RJ, Kian KH, Ali D, et al: pulmonary carcinoid tumor, Tanaffos 2002;1:51-56.

27. Todd TR, Cooper JD, Weissberg D, et al. Bronchial carcinoid tumors: twenty years experience. JThoracic Cardiovasc Surg 1980 79:532-35.

28. Ronchod M, Levine GD Spindle-cell carcinoid tumors of the lung: a clinicopathological study of 35 cases. Am U Surg Pathol 1980 4:315-30 .

29. Ismail C, Salih T, Irfan et al. Surgery in bronchial carcinoid: experience with 83 patients Eur J Cardiothoracic Surg 2002 21:883-887.

30. Elhassani NB: Bronchial carcinoid tumors, Ann Saudi med 1988 8:35. 